\title{
An integrated analysis of the Down Syndrome trajectory: Could implicit bias be driving Trisomy 21's phenotypes?
}

\author{
Robin Lynn Treptow*1 \\ ${ }^{1}$ Doctoral Student, Infant and Early Childhood Development Program in the School of Leadership Studies, Fielding Graduate University, Santa Barbara, California, \\ USA; Adjunct Faculty, Masters of Science in Psychology Program, Divine Mercy University, Arlington, Virginia, USA
}

\begin{abstract}
Implicit bias is bias in opinion and/or actions resulting from subtle thought processes that operate without overt control and outside conscious awareness. Evidence for implicit bias against persons with Trisomy 21 (i.e, Down Syndrome) is accruing; recent studies show such bias extends towards children with the diagnosis. It may occur in medical settings with adults and children with Trisomy 21 as well. Outward views of the Trisomy 21 population (e.g., positive expressions) often do not match internal ones (e.g., negative perceptions), even among professionals. To stimulate scholarly discussion about implicit bias and its potential epigenetic impact on persons with Trisomy 21, this integrated review knits together historical mindsets about Trisomy 21 with emerging research, theory, and intervention. Rooted in the implicit bias and Trisomy 21 literatures, the narrative depicts theorized developmental impacts of negative implicit bias on Trisomy 21 phenotypes. The perspective is that negative implicit bias forces the human psyche into a downward spiraling developmental cascade, crushing emotion-driven intellect. Negative implicit biases about Trisomy 21 have potency to derail development's typical course in infants, children, or even adults with this diagnosis. Reshaping these negative biases around Trisomy 21 into positive predictions can build resilience that turns the course towards normative development for individuals in this group. Recommendations drawn from this Trisomy 21 narrative are provided.
\end{abstract}

\section{Introduction}

Trisomy 21 (i.e., Down's Syndrome or Down Syndrome ${ }^{1}$ ) is a chromosomal disorder presumed to cause low intelligence. A genetically linked medical diagnosis, Trisomy 21 is marked by the presence of a triad, rather than a dyad, of the $21^{\text {st }}$ chromosome. Its associated effects of cognitive delay have long been viewed as inescapable putting prenatally diagnosed infants at higher risk for induced abortion [1-7]. Moreover, persons with Trisomy 21 who encounter negative biases about their potential (i.e., low expected intelligence or level of functioning) are at a developmental disadvantage due to the psychological cost of this stigma [8].

The purpose of this narrative review is to explore historical and emerging paradigms surrounding persons with Trisomy 21, predominantly in the context of implicit and explicit attitudes. It is argued that Trisomy 21 is principally a socially precipitated condition rather than the genetically driven disorder it is almost universally believed to be. First, historical ideas around Trisomy 21 are explored, including genetic and environmental propositions. Second, racially motivated implicit and explicit attitudes about persons with Trisomy 21 are investigated, including those towards children. Third, an alternative Trisomy 21 narrative dissociated from genetic predictions is explored. Finally, research and intervention recommendations are drawn from this newly created Trisomy 21 narrative.

\section{Bias-related definitions}

Before exploring implicit bias and racial prejudices in the context of Trisomy 21, basic terminology will be defined.

${ }^{1}$ Named for a physician named John Langdon Down who described a set of children with common features who were distinct from other children with mental retardation.

\section{Racism}

Racism is making up one's mind about another person's potential using observable physical traits (e.g. facial features, skin color, body morphology; race, 2017; racism, 2017) [9,10].

\section{Implicit bias}

Negative implicit bias is unfairness in judgment and/or behavior caused by subtle thought processes that operate outside overt control and without awareness [11,12].

\section{Implicit versus explicit attitude}

Judgments of which people are aware (i.e., explicit attitudes) often differ from their unconscious ones [11,12]. This means that people may believe they think positively about certain groups of people, but they really hold prejudices again those individuals-often based on specific traits (e.g., physical features, disability).

\section{Evidence for genetically based beliefs of poorer outcomes in Trisomy 21}

The belief that individuals with Trisomy 21 have less cognitive potential is long-standing. Bases for this deduction have as a rule been stoically genetic despite its risks $[6,13,14]$. In 1990 Wisniewski

Correspondence to: Robin Lynn Treptow ,1407 Park Garden Road, Great Falls, Montana 59404, USA, Tel: 1+406-899-1548; Fax: 1+805-687-6559; E-mail: rtreptow@email.fielding.edu

Key words: developmental cascade, epigenetic impact, implicit bias, resilience, selffulfilling prophecy, trisomy 21

Received: May 14, 2017; Accepted: June 05, 2017; Published: June 08, 2017 
wrote, '[a]ll Down syndrome (DS) children [emphasis added] have developmental brain abnormalities' (P.274); he suspected a genetic cause [15]. Borthwick (1996), conversely, doubted that genes dictated Trisomy 21's universally observed poorer cognitive outcomes; he suspected widespread social processes instead [16].

'If one were to add to those factors the further stipulations that the group under study was, even now, regarded as utterly and unquestionably intellectually inferior, that it has been regarded as inferior universally and in all cultures, that individuals in the group are regarded as inferior even by their own families and in relation to their own siblings, that the supports of a common culture are entirely absent, that not only their formal education but their basic instruction had historically been rudimentary or non-existent and that no positive role models for them have ever existed, one would expect a larger difference still; and one would be describing people with [Trisomy 21] [emphases added]' (P.404) [?].

\section{Genetics control Trisomy 21 debate}

Despite the aforementioned gene versus environment debate, genetics dominated studies of Trisomy 21 for decades. Hypotheses that environmental factors such as prejudice and discrimination could explain racial differences in measured intelligence were never applied to people with Trisomy 21. Borthwick (1996) framed 'this failure of imagination' (P.403) as resulting from unspoken limits on scholarly discourse in the field of intellectual disability [16]. In that era a 'neurodevelopmental disorder like Down syndrome (DS) was a recipe for cognitive difficulties often deemed insurmountable' [17]. Even today, epigenetic researchers ascribe Trisomy 21's widespread phenotypes to complex genetically driven factors (e.g., DNA methylation and post-translational histone modifications [18].

\section{Environmental hypotheses about Trisomy 21}

Two decades past Borthwick (1996) and Wisniewski's (1990) competing claims, attitudes about intelligence in persons with Trisomy 21 have become more accommodating of a variety of cognitive outcomes [15,16]. Roberts and Richmond (2015) doubted whether cognitive deficits linked to Trisomy 21 exist at birth [19]. Karmiloff-Smith et al. (2012; 2016) implied subtle differences in early environments affect immediate and long-term outcomes for this group [20-22].

'One question to emerge from cross-syndrome comparisons is why the positive effects of high SES are not greater in families with infants with genetic disorders. Unlike children from low SES environments, many children with neurogenetic syndromes are well-nourished, grow up in a caring environment, receive considerable cognitive stimulation, and do not suffer the physical and mental abuses that exist in some contexts of early social adversity. Therefore, why do such positive environments not compensate for genetic vulnerabilities?' [20]

Research in epigenetic therapies-or epigenetic alterations to genes to reverse phenotypic outcomes that theoretically result from gene expression-is underway [18]. Cognitive assessment measures designed specific to the Trisomy 21 population are surfacing and interventions that target key features of the Trisomy 21 phenotype (e.g., language difficulties) are taking root [17]. Data builds to show that epigenetic markers, including those crucial to memory formation or that drive synaptic plasticity, are reversible [18]. As pessimism entrenched in genetic rigidity fades, the notion that persons with Trisomy 21 are intellectually capable materializes.

\section{Racially precipitated implicit bias towards persons with Trisomy 21}

Despite this changing climate with its higher expectations, one facet of Trisomy 21 racism-tinged bias must be looked at more closely. Bias or stigma surrounding Trisomy creates barriers to thriving (Green, 2003) and may lead to prenatal termination or postnatally truncated care $[2,5,6,8,13,23]$.

' $[a] s$ they may have a negative impact on the lives of people with intellectual disabilities, implicit attitudes merit increased attention in research and interventions in the intellectual disabilities field.' (P.1) $[11,12]$

\section{How racism relates to Trisomy 21}

Causative analyses of the Trisomy 21 developmental course are tied to racism's purview. Facial and bodily features (e.g., low tone, protruding tongue, bilateral linear creases across both palms, smaller upturned eyes) flag the syndrome early on (Roper \& Reeves, 2006) [14]. Genome to phenome pathways ostensibly create the syndrome labeled for Dr. Down.

'DS [Down Syndrome] is a product of genetic effects on different cells, structures, and functions throughout development, many of which may have cascading effects to produce clinically observed phenotypic end points in a given individual with trisomy 21 . These are obviously not independent concepts; any "developmental" perturbation derives from alteration of some function in a developing cell' [14].

Deliberations about how genes in Trisomy 21 play out as observed structure and behavior are complex. Genetically steeped assumptions permeate even cutting-edge research climates aimed towards better outcomes for persons in this group [18].

'without knowledge about genetics and neurobiology, [Dr. Down] was ahead of his time by postulating "that there can be no doubt that these ethnic features are the result of degeneration [emphases added]."' (P.72)

Of interest, each primary Trisomy 21 phenotype 'may also occur in euploid individuals [those having the normal number of chromosomes for the human species]' [14]. Also, Karmiloff-Smith et al. (2016) described the Trisomy 21 brain 'as developing relatively normally during the first few months postnatally (P. 2; Roberts \& Richmond, 2015) $[19,21]$.

\section{Trisomy 21 diagnosis based on racial features}

Though also present in persons without the diagnosis, physical traits associated with Trisomy 21 seem to give an impression that all is not medically satisfactory [14]. Presumptive clinical diagnoses based on dysmorphic features (i.e., differently formed body structures; dysmorphic, 2017; dysmorphic feature, 2011) often precede genetic testing in cases of chromosomal disorders like Trisomy 21 [24-26]. In a hypothetical vignette study of infants who lacked atypical bodily features physicians were highly unlikely to refer for genetic testing even in cases of global developmental delays (i.e., in only $5 \%$ of cases [27]. Thus, a group of corporeal characteristics that differ from typical patterns appear to drive medical concern about genomic threats to cognitive development.

\section{Social and historical linkages to Trisomy 21 phenotypes}

Societally promulgated historical links between observed phenotypes and Trisomy 21 genetic markers remain strong. Borthwick 
(1996) [16] tracked this correspondence early on: 'the complex relationship between racism and prejudice against people with Disability is illustrated by Dr. Down's use of the term 'mongolism" (P.403). While use of the terminology 'mongolism' as referent to Trisomy 21 has largely disappeared, clustered racial features still govern perceptions of the Trisomy 21 prototype [28]. It as if modern thinking about how children with this genomic make-up must be and behave stays permanently yoked to Dr. Down's early analysis. Many of these attitudes are outside of awareness, attributed to a concept called implicit bias $[11,12,28]$.

\section{Negative implicit bias and Trisomy 21}

Evidence accrues for implicit bias against persons with Trisomy 21 and others who have presumed cognitive disabilities [11,12,28]. Implicit bias is most often negative in content and may differ significantly from what persons think they believe about people in this group $[11,12,28]$. Studies on the whole show external attitudes (e.g., positive expressions) to be discrepant from internal ones (e.g., negative perceptions) towards individuals in the cognitive disabilities population $[11,12]$. Such biases extend downwards towards children who have Trisomy 21 and the inconsistencies have been found to exist even among health professionals $[11,28]$.

The paradigm of implicit bias related to cognitive disabilities is yet emerging and novel factors are being examined. Negative implicit bias towards persons with cognitive disabilities does not seem to be linked to social distance or emotional attitudes; nor does it vary according to the degree of contact participants have with persons who have intellectual disabilities [12]. Indeed, in Wilson and Scior's (2015) online survey, negative implicit attitudes were also not related to participants' education level, gender, or contact relationship [i.e., voluntary or involuntary] [12]. In contrast, explicit attitudes and social distance from persons with cognitive disabilities were associated with one or more of the aforementioned factors [12].

\section{Implicit bias and children with Trisomy 21}

Developmentally, it is disturbing that unconscious prejudices (i.e., implicit biases) extend even towards children who have stereotypic features linked to Trisomy 21. For instance, when the faces of children with Trisomy 21 were paired with positive or negative attributes, Enea-Drapeau et al. (2012) found positive explicit attitudes alongside negative implicit views of these children [28]. That is, respondents' outward stance implied they thought well of children who had Trisomy 21 while their hidden attitudes revealed a pejorative bias. Moreover, respondents' positive explicit attitudes were lower if faces had more stereotypic facial features. These authors declare:

'we also show that this [positive] bias can coexist with negative evaluations at the implicit level (with large effect sizes) [emphasis added], even among professional caregivers.' [28]

Similarly, Wilson and Scior (2015) found negative implicit attitudes towards persons with intellectual disabilities in their web-based study; implicit and explicit attitudes were not linked [12].

\section{Risk of implicit bias towards Trisomy 21 in medical settings}

Children with ethnic or racial features, including children with Trisomy 21, are at risk to encounter health provider initiated racially tinged implicit bias [21]. Children with Trisomy 21 have increased medical needs [29]. They encounter physicians (e.g., pediatricians, geneticists, cardiac specialists) who make diagnoses about their condition shortly after birth and often for many years thereafter [24,29]. They and their families frequently encounter health professionals e.g., in hospitals, clinics, and in home settings-considerably more often on average than other families and other children [29].

\section{A theory of how negative genetically based beliefs impact development}

Implicit biases against Trisomy 21 are culpable to impact the immediate and long-term development of children towards whom they are directed. These biases may have many sources: professional helpers, individuals in the wider society, or the parents themselves $[5,8,28]$. Contributors to a trajectory of implicit bias with its developmental risks will be discussed below.

\section{Implicit bias and early development}

Society has expectations about how babies ought to be; parents in every culture hope for health and well being for their newborns $[5,30,31]$. For instance, in the general context of infants diagnosed with disabilities, Hollins et al. (2010) elaborated.

Discovering that your newborn baby is not the hoped-for healthy baby, but has significant physical or mental impairments...is a significant source of physical, emotional, and spiritual distress for parents as well as siblings and the child's wider family.' (P.301) [5]. Foley (2006) likewise opined that families must grieve and accept an infant who is not the anticipated or desired child [30].

\section{Implicit bias model of Trisomy 21 phenotypes}

In the context of a trisomic genome for the $21^{\text {st }}$ chromosome, the logic of having expected a certain type of baby often narrows to focus on invariably negative prognoses and advice-giving [24,29]. Brave parents may stand against this projection, yet the diffident message permeates many cultures [24,31]. It is as if parents are not permitted to hope and dream like others once a Trisomy 21 diagnosis has been either suspected or confirmed.

'There is, therefore, a vital need for a more dynamic notion of environment (e.g., how having a neurodevelopmental disorder may subtly change the social, cognitive, linguistic, emotional, and physical environments in which the atypically developing child grows)' [20]

To change the insidious climate that nowadays surrounds Trisomy 21, downward spiraling developmental cascades must be turned upwards towards resilience $[1,18,32,33]$.

\section{Negative prophecies imbued in the genetic model}

Cautionary stances offered to parents regarding outcome in cases of Trisomy 21 are taken as status quo among medical providers [24,29]. Still, such subtle social messaging may undermine infants' development by disrupting parent-infant attachment [23].

'This scholarship demonstrates compellingly that it is rarely, if ever, the case that there is a significant main effect of genes on behavior. When one appreciates the dynamic nature of the developmental system, one recognizes the thorough coactions between genes and all other levels of organization within the system.' (P.339)

\section{Negative implicit bias conveyed via diagnosis}

Parents of newborns are ordinarily met with warm enthusiasm in hospital, yet that narrative course vacillates once Trisomy 21 is suspected [21]. Channels of communication around medical diagnosis and treatment are often complex [20,34]. 
'hypothesized that the moment that a parent is informed that their child has a genetic disorder, the parent's behavior subtly changes. As a result, the baby's responses within the dyadic interaction will also be subtly modified.' (P.17263) In discussing adult outcomes in individuals with Trisomy 21, Ball and Karmiloff-Smith (2014) likewise noted [35].

it is crucial to trace such cognitive-level outcomes back to their basic-level processes in early development and to consider their cascading effects over developmental time.' (P. 21)

Health providers may see stereotypic physical markers (i.e., a constellation of facial and bodily traits: hypotonia, extended tongue, bilateral linear creases across both palms, undersized upturned eyes). Perceiving these markers as indicative of Trisomy 21 could set the stage for providers' unconscious bias, thus nudging into play negatively charged developmental cascades [17,32,33]. Instinctive parent-infant dance steps become disrupted.

\section{Implicit bias and parent-infant interaction}

Should parents view the infant like any other baby, health providers who link Trisomy 21 to low potential may not know what to think about this degree of hopefulness. Trying to help, they may guide parents to grasp the provider's own reality of what the diagnosis means based on that provider's understanding and exposure to prior clinical cases or studies [24,29]. Parents then must use precious emotional resources to ponder and discard or keep this professional input (Figure 1).

Finding cognitive function in typically developing infants to be impacted by subtle variances in early mother-infant interaction, Karmiloff-Smith et al. (2012) asserted [20]. 'The developmental trajectories of those infants who grow up with genetic or environmental vulnerabilities are likely to be even more heavily influenced.' (P.17264)

If parents' energy to engage whole-heartedly with their infant is compromised due to professionals' well-meant counsel (i.e., doubts of the infant's ability), they are less likely to be attuned to the infant's subtler cues. A greater number of missed or unrepaired exchanges occur [36]. In this way, derailed social-cognitive neural networks can result if infant-to-parent cues are missed due to parents' distraction with health providers' opposing agendas [37].

\section{An alternative model of Trisomy 21 development}

Early expectations for infants with Trisomy 21 as well as perceptions of parents with optimistic outlooks in such cases may influence outcomes for this vulnerable group. Health providers' favorable clinical judgments around Trisomy 21 are of great consequence. Seeing the infant's potential as excellent, and its manner as engaged rather than filled with deficits and flaccid, might stir up families' nascent capacities to provide a nurturing 'holding space' for optimal infant vitality.

\section{Positive implicit bias negates downward spirals}

In short, health providers' encouraging attitudes towards the complex story line of Trisomy 21 may help bolster parents' attachment. Nurturing engagement may then stimulate these infants to thrive intellectually, referred to most succinctly in Stanley Greenspan and Selma Wieder's 'emotion-as-driver' model $[38,39]$. According to Greenspan, intelligence takes root in joint attention between parent and infant that progresses to circles of communication and shared meaning making over time.

A series of discrete yet overlapping functional emotional developmental capacities (FEDCs) form the core of back-and-forth communicative exchanges-stimulating infants' desire to engage with the environment around them. Shared attention, engaging the world, and interaction and communication are the basic FEDCs; they support shared problem-solving, meaningful use of ideas, and logical thinking [23,38-40].

'Genes do not exert an additive and independent-of-environment influence on behavior and development genes always function through interaction with the context contemporary developmental science places genes within a coacting, dynamic system of relations among the multiple levels of organization that comprise the ecology of human development.' (P.338) Parents and other caregivers are of chief importance in that milieu.

\section{Foundations for high level thought}

At the pinnacle of Greenspan's dynamic, interactive theory of intelligence lie three levels of thinking: multicausal, comparative and gray-area, and reflective; see Greenspan et al., 2010: P.75) [40]. These are highly complex levels of intellectual dialogue. If parents' energy to engage whole-heartedly with their infant remains unfettered due to professionals' grounded optimism (i.e., focus on the infant's potential), they are more likely to stay attuned to the infant's subtler cues. Fewer missed or unrepaired exchanges result [36].

In this latter case, the infant's chance to reach advanced cognitive capacities over developmental time is markedly enhanced. As Edgin et al. (2015) articulated the neuroconstructivist view [17].

Cognitive-level differences in older children and adults with neurodevelopmental disorders must be traced back to their more basic precursors in infancy and early childhood.' (P.2)

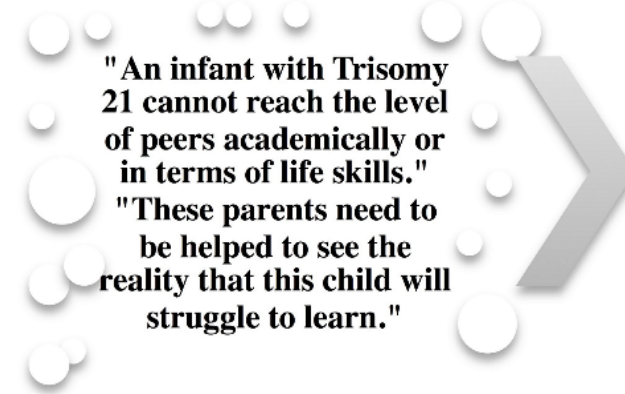

Cautious attitudes

\begin{abstract}
Tacit and overt negative messages are transferred to the parents who must process through them to discard them or accept them as truth; this task takes away from psychic energy needed for spontaneous, efficacious bonding.
\end{abstract}

\section{Spoken and unspoken messages}

\author{
The attachment bond is \\ subtly disrupted: \\ resulting in a different \\ quality of attachment. \\ These tacit rifts in the \\ love dance interrupt the \\ flow of intelligence \\ (Greenspan, 1989).
}

\section{Parent-infant attachment is subtly disrupted}

Figure 1. Parents then must use precious emotional resources to ponder and discard or keep this professional input 
Robust social-cognitive neural networks, which build cognitive capacity, are most likely to result if infant-to-parent cues are attended to in an early and supportive context from the moment of birth [37].

\section{Summary and conclusion}

Recall that Roberts and Richmond (2015) had 'question[ed] the assumption that cognitive deficits associated with this genetic abnormality [Trisomy 21] are in fact present from birth' (P.415) [19]. Karmiloff-Smith et al. (2012) likewise deduced [20].

'is it possible that this finding [i.e., lack of improved outcomes for infants with congenital conditions even in nurturing families] is [due to] the fact that early environments differ in more subtle ways than is commonly realized [emphasis added]?' (P.17263)

\section{Looking at early implicit bias is crucial}

For these reasons, as well as many others, the social concomitant of implicit bias as trigger to perceived imminent cognitive dysfunction in Trisomy 21 cases deserves inquiry. The World Association of Infant Mental Health (WAIMH, 2016) advocates that professionals honor parents while serving infants [41]. But if negative predictions guide health providers' discourse then hopeful, energetic parents could be dissuaded from spontaneous engagement in the affective dance theorized by Greenspan to drive thinking competencies [38-40].

As described in Edgin et al. (2015) [1]. '[effective] interventions should aim to influence global neural organization in ways that help to normalize patterns of connectivity and establish more mature brain networks, again starting as early as possible in infancy.' (P.2) Ball and Karmiloff-Smith (2014) similarly asserted [35].

'to understand ontogenetic development...it is vital to trace developmental trajectories across time to assess progressive change from infancy onwards at the genetic, neural, cognitive, and behavioural levels, including the role of the environment [emphases added]' (P.19)

The idea is that parents ought to keep ahold of their vision of the 'idealized child' in cases of a Trisomy 21 diagnosis rather than accepting an alternative, less optimal scenario as is now both advised and expected [30].

\section{Final remarks on the early impact of implicit bias}

Social messages around a Trisomy 21 diagnosis alter what the parent-infant interaction looks like, which in turn may affect infants' developmental trajectories [21]. Development, learning, and neurological growth take root in this subtly, yet meaningfully, changed environment [37]. Divergent patterns of interpersonal engagement continue throughout childhood as social circumstances, including the influence of peers, evolve across the developmental stages [37,42].

'behavior genetics offers science and the societal audience for science not only simpler (albeit egregiously inept) methods than the ones that are used by developmental systems-oriented scholars... we must move beyond the important but still relatively simplistic and largely sterile study of $\mathrm{G} \times \mathrm{E}$ and address the more complex but necessary task of the analysis of the developmental system.' (P.339).

A better grasp of implicit bias around disability in the earliest days of an infant's life is therefore crucial to altering expected outcomes for children and adults with a Trisomy 21 diagnosis. Karmiloff-Smith et al. (2012) summed it up best [20].

'If such subtle differences in early mother-infant interaction in TD [typically development] infants growing up in nonadverse environments can have such effects on the timing of cognitive milestones, the developmental trajectories of those infants who grow up with genetic or environmental vulnerabilities are likely to be even more heavily influenced.' (P. 17264)

\section{Future recommendations}

Infants with Trisomy 21 who face a world filled with negative expectations of their potential (i.e., lower intelligence and day-to-day functioning) are at a developmental disadvantage [21,35]. Implicit bias's impact has been shown in this limited overview, yet more data about that dynamic needed. For instance, how early can this bias be identified? What processes (e.g., seeing a cluster of facial and bodily traits) set the prejudice into play? Which persons first impact the bias's course? Does the stigma extend to caregivers like an infant's parents?

Innovative research is essential to ascertaining pathways to resilience in cases of Trisomy 21 [1,33]. To identify, and rewire, Trisomy 21's epigenetic pathways towards thriving and intellectual achievement will take persistence and creativity. Qualitative vignette studies may fit this line of investigation. Researchers might use such studies to explore rigid mindsets about the innate potential of persons with Trisomy 21 among professionals (e.g., health providers, early intervention workers) as well as in the general public [43,44]. Vignette studies, alone or joined with implicit association tests (IATs), might also look at bias by varied social groups towards newborn infants who present with features stereotypic of Trisomy $21[27,45]$.

Education programs for health providers and educators should examine the dynamics of negative self-fulfilling prophecy, and how it can be triggered by implicit bias. Social decision-making factors in this context must be explored [46,47]. Training programs might give the parents of children with Trisomy 21 valuable tools to stimulate their children's intellectual growth. For example, tutorials that look at models of universal learned helplessness self-efficacy; emotiondirected cognition ; and/or self-fulfilling prophecy could help families get past the hurdles built by implicit bias [38-40,48-50].

\section{Authorship and contributorship}

Robin Lynn Treptow is responsible for the content of this manuscript; she wrote it and provided all the intellectual material contained within it. Any errors or oversights are attributable to the author alone.

\section{Acknowledgements}

The author is indebted to Joy Voyles Browne, Ph.D., PCNS, IMH-E (IV), for her intellectual and editorial input during prior iterations of this manuscript as excerpted from a dissertation concept paper prepared under her tutelage.

\section{Funding information}

No funding was obtained for the writing of this manuscript.

\section{Competing interest}

The author declares future financial competing interests related to this manuscript in that she is developing a consultation model for families of children with disabilities based on several of this article's premises. The author further declares that she has a non-financial competing interest related to this manuscript in that she has a son with Trisomy 21 and ideologically questions whether the dominant genetic paradigm truly explains that medical diagnosis's common phenotypes. 
The author declares that she has no other competing financial or nonfinancial interests related to this manuscript.

\section{References}

1. Edgin JO, Clark CAC, Massand E, Karmiloff-Smith AD (2015). Building an adaptive brain across development: Targets for neurorehabilitation must begin in infancy. Front Behav Neurosci 9: 1-15. [Crossref]

2. de Graaf G, Buckley F, Skotko BG (2015) Estimates of the live births, natural losses, and elective terminations with Down syndrome in the United States. Am J Med Genet A 167: 756-767. [Crossref]

3. Hans JD, Kimberly C (2014) Abortion attitudes in context: a multidimensional vignette approach. Soc Sci Res 48: 145-156. [Crossref]

4. Hawkins A, Stenzel A, Taylor J, Chock VY, Hudgins L (2013). Variables influencing pregnancy termination following prenatal diagnosis of fetal chromosome abnormalities. $J$ Genet Couns 22: 238-248. [Crossref]

5. Hollins S, Woodward S, Hollins K (2010) Parenting an infant with a disability: Parenthood and Mental Health: A Bridge between Infant and Adult Psychiatry 301310 .

6. Phelan JC, Link BG, Feldman NM (2013) The genomic revolution and beliefs about essential racial differences a backdoor to eugenics? Am Socio Rev 78: 167-191. [Crossref].

7. Skotko BG (2009) With new prenatal testing, will babies with Down syndrome slowly disappear? Arch Dis Child 94: 823-826. [Crossref]

8. Green SE (2003) "What do you mean 'what's wrong with her?"': stigma and the lives of families of children with disabilities. Soc Sci Med 57: 1361-1374. [Crossref]

9. In Merriam-Webster Online. Retrieved May 12, 2017 race [Def. 3.3b]

10. In Merriam-Webster Online. Retrieved from May 12, 2017. Racism [Def. 1].

11. Wilson MC, Scior K (2014) Attitudes towards individuals with disabilities as measured by the Implicit Association Test: A literature review. Res Dev Disabil 35: 294-321. [Crossref]

12. Wilson MC, Scior K (2015) Implicit attitudes towards people with intellectual disabilities: Their relationship with explicit attitudes, social distance, emotions and contact. PloS One 10: e0137902. [Crossref]

13. Antommaria AM (2006) 'Who should survive? One of the choices on our conscience' Mental retardation and the history of contemporary bioethics. Kennedy Inst Ethic $J 16$ : 205-224. [Crossref]

14. Roper RJ, Reeves RH (2006) Understanding the basis for Down syndrome phenotypes. PLoS Genet 2: e50. [Crossref]

15. Wisniewski KE (1990) Down syndrome children often have brain with maturation delay, retardation of growth, and cortical dysgenesis. Am J Med Genet Suppl 37: 274 281. [Crossref]

16. Borthwick C (1996) Racism, IQ and Down's syndrome. Disabil Soc 11: 403-410.

17. Edgin JO, Clark CAC, Massand E, Karmiloff-Smith AD (2015). Building an adaptive brain across development: Targets for neurorehabilitation must begin in infancy. Front Behav Neurosci 9: 1-15. [Crossref]

18. Dekker AD, De Deyn PP, Rots MG (2014) Epigenetics: the neglected key to minimize learning and memory deficits in Down syndrome. Neurosci Biobehav Rev 45: 72-84. [Crossref]

19. Roberts LV1, Richmond JL (2015) Preschoolers with Down syndrome do not yet show the learning and memory impairments seen in adults with Down syndrome. Dev Sci 18: 404-419. [Crossref]

20. Johnson TJ, Winger DG, Hickey RW, Switzer GE, Miller E, et al. (2017) Comparison of physician implicit racial bias toward adults versus children. Acad Pediatr 17: 120126. [Crossref]

21. Karmiloff-Smith A, D’Souza D, Dekker TM, Van Herwegen J, Xu F, et al. (2012) Genetic and environmental vulnerabilities in children with neurodevelopmental disorders. Proc Natl Acad Sci USA, 109: 17261-17265. [Crossref]

22. Karmiloff-Smith A, Al-Janabi T, D'Souza H, Groet J, Massand E, et al. (2016) The importance of understanding individual differences in Down syndrome. F1000Research 5: 1-10. [Crossref]

23. Kodadek MP, Feeg VD (2002) Using vignettes to explore how parents approach endof-life decision making for terminally ill infants. Pediatr Nurs 28: 333. [Crossref]
24. Dent KM, Carey JC (2006) Breaking difficult news in a newborn setting: Down syndrome as a paradigm. Am J Med Genet C Semin Med Genet 142C: 173-179. [Crossref]

25. Dysmorphic (2017) In Merriam-Webster Medical Dictionary Online. Retrieved May $13,2017$.

26. Dysmorphic feature. Segen's Medical Dictionary (2011) Retrieved May 13, 2017.

27. Tarini BA, Zikmund-Fisher B J, Saal HM, Edmondson L, Uhlmann W R (2015) Primary care providers' initial evaluation of children with global developmental delay: a clinical vignette study. J Pediatr 167: 1404-1408. [Crossref]

28. Enea-Drapeau C, Carlier M, Huguet P (2012) Tracking subtle stereotypes of children with Trisomy 21: From facial-feature-based to implicit stereotyping. PLoS One 7 e34369. [Crossref]

29. Skotko BG, Davidson EJ, Weintraub G S (2013) Contributions of a specialty clinic for children and adolescents with Down syndrome. Am J Med Genet A 161A: 430- 437. [Crossref]

30. Foley GM (2006) The loss-grief cycle: Coming to terms with the birth of a child with a disability. In GM. Foley, JD Hochman (Eds.), Mental Health in Early Intervention: Achieving Unity in Principles and Practice 227-243. Baltimore, Maryland: Brookes Publishing Co.

31. Rogoff B (2003) The Cultural Nature of Human Development. New York, NY Oxford University Press.

32. Masten A (2014) Ordinary magic: Resilience in development. New York: Guilford Press.

33. Masten AS, Cicchetti D (2010) Developmental cascades. Dev Psychopathol 22: 491 495. [Crossref]

34. Zwaanswijk M, Tates K, van Dulmen S, Hoogerbrugge PM, Kamps WA, et al (2011) Communicating with child patients in pediatric oncology consultations: a vignette study on child patients', parents', and survivors' communication preferences. Psychooncology 20: 269-277. [Crossref]

35. Ball G, Karmiloff-Smith A (2014). Why development matters in neurodevelopmental disorders. In J. van Herwegen \& D. Riby (Eds.). Neurodevelopmental Disorders: Research Challenges and Solutions, 19-33. Research Methods in Developmental Psychology: A Handbook Series. London, UK: Psychology Press.

36. Tronick E, Beeghly M (2011) Infants' meaning-making and the development of mental health problems. Am Psychol 66: 107-119. [Crossref]

37. Iarocci G, Yager J, Rombough A, McLaughlin J (2008) The development of socia competence among persons with Down syndrome: From survival to social inclusion. Int Rev Res Ment Ret 35: 87-119.

38. Greenspan SI (1989) The Development of the Ego: Implications for Personality Theory, Psychopathology, and the Psychotherapeutic Process. Madison, WI: International Universities Press.

39. Greenspan SI, Wieder S (2008) The interdisciplinary council on developmental and learning disorders diagnostic manual for infants and young Children-An overview. $J$ Can Acad Child Adolesc Psychiatry 17: 76-89. [Crossref]

40. Greenspan SI, Greenspan NT, Lodish R (2010) The Learning Tree: Overcoming Learning Disabilities from the Ground Up. Cambridge, MA: De Capo Press.

41. World Association for Infant Mental Health (WAIMH; 2016). Position Paper on Infant Rights. Perspect Infant Ment Health, Winter-Spring.

42. Lerner R M (2006) Another nine-inch nail for behavioral genetics! Hum Dev 49: 336 342 .

43. Jackson M, Harrison P, Swinburn B, Lawrence M (2015) Using a Qualitative Vignette to Explore a Complex Public Health Issue. Qual Health Res 25: 1395-1409. [Crossref]

44. Keane D, Lang A R, Craven MP, Sharples S (2012) The use of vignettes for conducting healthcare research. In: V. G. Duffy (Ed.), Advances in Human Aspects of Healthcare (451- 460). San Francisco, United States of America, 21- 25 July 2012. Boca Raton: CRC Press.

45. Wissow LS, Zafar W, Fothergill K, Ruble A, Slade E (2016) Using vignettes to assess contributions to the work of addressing child mental health problems in primary care. BMC Health Serv Res 15: 1-10. [Crossref]

46. McCleary N, Ramsay CR, Francis JJ, Campbell MK, Allan J (2014) Perceived difficulty and appropriateness of decision making by General Practitioners: A systematic review of scenario studies. BMC Health Serv Res 14: 1-13. [Crossref] 
Treptow RL (2017) An integrated analysis of the Down Syndrome trajectory: Could implicit bias be driving Trisomy 21's phenotypes?

47. Müller-Engelmann M, Donner-Banzhoff N, Keller H, Rosinger L, Sauer C, et al. (2013) When decisions should be shared: A study of social norms in medical decision making using a factorial survey approach. Med Decis Making 38-47. [Crossref]

48. Abramson LY, Seligman, ME,Teasdale JD (1978) Learned helplessness in humans: Critique and reformulation. J Abnorm Psychol 87: 49-74. [Crossref]
49. Sternberg E, Critchley S, Gallagher S, Raman VV (2011) A self-fulfilling prophecy: linking belief to behavior. Ann NY Acad Sci 1234: 98-99. [Crossref]

50. Wurm S, Warner LM, Ziegelmann JP, Wolff JK, Schüz B (2013) How do negative self-perceptions of aging become a self-fulfilling prophecy? Psychol Aging 28: 10881097 [Crossref]

Copyright: (C2017 Treptow RL. This is an open-access article distributed under the terms of the Creative Commons Attribution License, which permits unrestricted use, distribution, and reproduction in any medium, provided the original author and source are credited. 